\title{
Hot topics in kidney cancer 2010
}

\author{
Axel S. Merseburger · Börje Ljungberg • \\ Christian Doehn
}

Received: 5 April 2010/Accepted: 13 April 2010/Published online: 5 May 2010

(C) Springer-Verlag 2010

The incidence of renal cell carcinoma has increased in recent years, becoming the third most common urologic tumour. This topic issue of the World Journal of Urology provides an updated overview on epidemiology with perspectives on primary prevention by Weikert et al., proceeding from there to an update of imaging and diagnosis by Müller-Lisse.

With regard to imaging, it is important for the surgeon to know the reliability of radiological findings in correlation with pathological outcomes. The article by Hong et al. covers this important issue in detail.

In some cases with renal lesions of unknown dignity, biopsies are helpful in the preoperative differential diagnosis. In this study by Chyhrai et al., the benefit of multi-colour interphase fluorescence in situ hybridization on fine-needle core biopsies in uncertain renal masses is

\footnotetext{
A. S. Merseburger

Department of Urology and Urologic Oncology,

Medical School of Hanover [MHH],

Carl Neuberg Str. 1, 30625 Hanover, Germany

B. Ljungberg

Department of Surgical and Perioperative Sciences,

Umeå University, 90185 Umeå, Sweden

C. Doehn $(\bowtie)$

Department of Urology,

University of Lübeck Medical School,

Ratzeburger Allee 160, 23538 Lübeck, Germany

e-mail: doehn@medinf.mu-luebeck.de
}

described. Augmenting prognosis and treatment, Remzi et al. describe the treatment of small renal lesions and possible benign behaviours.

Laparoscopic radical nephrectomy is a feasible procedure in treating $\mathrm{T} 1$ and $\mathrm{T} 2$ tumours and possibly $\mathrm{T} 3$ or even more advanced lesions. However, every novel technique has to be evaluated and compared to the standard open procedure in terms of oncological long term outcome. Chen et al. evaluate this question for $\mathrm{T} 1 / \mathrm{T} 2$ renal malignancies in a large cohort of patients.

Prognostic factors following treatment in localised and advanced disease are of importance, but, unfortunately, the impact and interaction is often unclear. Waalkes et al. demonstrate in a multivariate analysis of a large cohort of patients that the urinary collecting system (UCS) invasion is not an independent prognostic factor in renal cell carcinoma.

The treatment of patients with renal cell cancers has evolved substantially during the last decade, mainly due to the introduction of new targeted agents to cure metastatic disease. Less than a decade ago, the progression-free expected survival of patients with metastatic disease was about 3-5 months, with immune therapy. Since the introduction of tyrosine kinase inhibitors, the relapse rate has more than doubled. These important changes in the treatment of advanced renal cancer are discussed in three articles.

Finally, Gruschwitz et al. address the role of genetic differentiation for the improvement of histopathological classification of adrenal gland tumours. Nowadays, genetic tests with fine-needle biopsies could be offered in the routine diagnostics of adrenal specimens of unknown origin.

We would like to take this opportunity to sincerely thank Professor Dr. Martin Burchardt for inviting us as 
guest editors for this issue of Hot Topics in Kidney Cancer, 2010 for the World Journal of Urology.

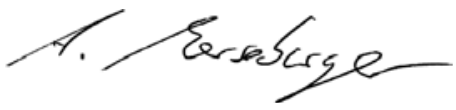

Axel S. Merseburger, MD, PhD

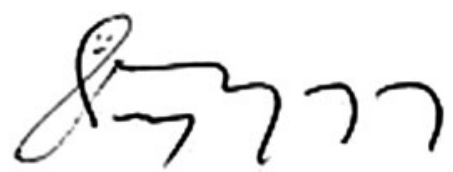

Börje Ljungberg, $\mathrm{MD}, \mathrm{PhD}$

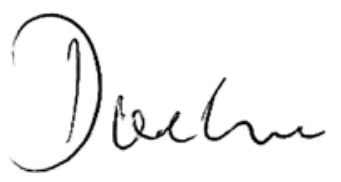

Christian Doehn, MD, PhD 